\title{
Effective Field Theory and High-Precision Calculations of Nuclear Electroweak Processes*
}

\author{
Kuniharu Kubodera ${ }^{1}$ and Mannque Rho ${ }^{2}$ \\ ${ }^{1}$ Department of Physics and Astronomy, \\ University of South Carolina, \\ Columbia, SC 29208, USA \\ 2 Institut de Physique Théorique, CEA Saclay, \\ 91191 GIf-sur-Yvette Cédex, France, \& \\ Department of Physics, Hanyang University, \\ Seoul 133-791, Korea
}

\begin{abstract}
High-precision calculations of electroweak processes in light nuclei are of great importance for multiple reasons. Gerry Brown together with Dan-Olof Riska published in 1972 a famous calculation on radiative capture of a thermal neutron on a proton, and their work was a precursor of subsequent great developments in high-precision calculations of electroweak processes in light nuclei. The application of effective field theory to nuclear systems is a prominent example of these developments. We present an overview of it with main emphasis placed on those topics in which we ourselves have been involved.
\end{abstract}

${ }^{*}$ To appear in Festschrift for Gerry Brown, ed. Sabine Lee (World Scientific, Singapore) 


\section{Introduction}

In 1972 Dan-Olof Riska and Gerry Brown published their celebrated calculation [1] on radiative capture of a thermal neutron on a proton: $n p \rightarrow \gamma d$. Until their work appeared, the $\sim 10 \%$ discrepancy between the experimental and theoretical values of the radiative $n p$ capture rate had been a persistent puzzle. Dan-Olof and Gerry demonstrated that this discrepancy could be removed by including the contributions of the exchange currents derived by Chemtob and Rho $[2],{ }^{1}$ and this was the first convincing case that pointed to the existence of the exchange currents in nuclei. The 1972 Riska-Brown paper was a precursor of subsequent important developments in high-precision calculations of electroweak processes in light nuclei. On the occasion of Gerry's 85th birthday, we would like to give a succinct survey of these developments; we shall concentrate here mostly on those topics we ourselves have worked on, and so this note is not meant to be a comprehensive survey of the field.

High-precision calculations of electroweak processes in light nuclei are of great importance for multiple reasons. First of all, light nuclei, for which nuclear manybody-problem aspects are relatively well controlled, are expected to provide clean testing grounds for our understanding of the behavior of the hadronic electroweak currents in nuclei. In this connection, we should emphasize that the recent progress in formalisms and numerical techniques for solving the multi-nucleon Schrödinger equation has reached such a level [3] that the wave functions of low-lying levels for light nuclei can now be obtained practically with no approximation (once the validity of a given nuclear Hamiltonian is assumed). This situation frees us from the "usual" nuclear physics complications that arise as a result of truncating the nuclear Hilbert space down to certain model space (such as shell-model configurations, cluster-model trial functions, RPA, etc.).

We should also mention that nuclear electroweak processes in light nuclei are of particular importance in connection with astrophysics and particle physics. They appear in many astrophysical phenomena, prominent examples being stellar evolution (including solar nuclear fusion), big-bang nucleo-synthesis, supernova nucleosynthesis, etc. Furthermore, in terrestrial experiments designed to detect astrophysical neutrinos, weak-interaction reactions on light nuclei play important roles. It is also to be noted that neutrino-nucleus reactions feature importantly in neutrinooscillation experiments, one of the most hotly pursued frontiers in particle physics. Reliable information on the cross sections for these processes is obviously in great demand. However, for many of the processes in question, it is extremely difficult, or even impossible at present, to experimentally measure their cross sections. We must therefore totally rely on theoretical estimates, and this implies that we need a theoretical framework that allows us to arrive at estimates with reliable error assessments attached. Fortunately, the past two decades have witnessed significant progress toward this goal.

\footnotetext{
${ }^{1}$ see M. Rho, in this volume.
} 
Recent developments in high-precision calculations of electroweak reactions in light nuclei encompass two lines of studies. One is the elaboration and expansion of the phenomenological potential model, which has come to be known as the standard nuclear physics approach (SNPA). Another line is the successful application of effective field theory (EFT) to nuclear physics. In the sense that the former is phenomenological in nature whereas the latter is based on a systematic expansion that is formally in conformity with the tenets of EFT, these two approaches are distinct and, perhaps because of this, it happens rather often that practitioners of one approach (SNPA or EFT) ignore the existence of and progress in the other approach. In our view, however, this does not seem an ideal or even productive situation. As discussed below, the forte of EFT is that it gives a clear-cut guiding principle on how to tame short-range physics. But, as far as long-range nuclear correlations are concerned, EFT does not necessarily lead to improvement over what has already been achieved in SNPA. And, as we will illustrate with concrete examples later, the high-precision calculation of an observable often requires that both short-range physics and long-range nuclear correlations are under control. So, at least at present, it appears to be a reasonable strategy to take advantage of the availability and the power of both SNPA and EFT while assuring, as best one can, consistency between the two. This viewpoint led us to propose the "more effective" effective field theory (MEEFT) approach, also called EFT* ${ }^{*}$ It should be emphasized here that Tae-Sun Park has played a particularly important role in the developments of this approach. The nature of EFT limits us to consider only those processes which involve relatively low energies and momenta but, within this limitation, MEEFT has registered remarkable successes in giving reliable estimates for electroweak transition rates of current interest. We wish to describe here some of these results; primarily we shall be concerned with the following processes:

$$
\begin{array}{ll}
p p \text {-fusion: } & p+p \rightarrow d+e^{+}+\nu_{e} \\
\nu \text { - } d \text { reactions: } & \nu_{e}+d \rightarrow e^{-}+p+p, \quad \nu+d \rightarrow \nu+n+p \\
\mu \text { - } d \text { capture: } & \mu^{-}+d \rightarrow \nu_{\mu}+n+n \\
\text { Radiatice } \pi \text {-capture: } & \pi^{-}+d \rightarrow \gamma+n+n . \\
\text { Tritium } \beta \text {-decay: } & t \rightarrow{ }^{3} \mathrm{He}+e^{-}+\bar{\nu}_{e} \\
\mu-{ }^{3} \text { He capture: } & \mu^{-}+{ }^{3} \mathrm{He} \rightarrow \nu_{\mu}+t \\
\text { Hep reaction } & { }^{3} \mathrm{He}+p \rightarrow{ }^{4} \mathrm{He}+\nu_{e}+e^{+} \\
\text {Hen reaction } & { }^{3} \mathrm{He}+n \rightarrow{ }^{4} \mathrm{He}+\gamma
\end{array}
$$

This list may look like a haphazardly picked collection but, as will be explained below, they are interrelated in the context of demonstrating the important progress achieved in MEEFT. Specific motivations for considering these reactions will be described later for each case as the individual processes are discussed. Just to set the stage, however, we make some remarks here on the following three processes: $p p$-fusion, Hep and the $\nu d$ reaction. 
The $p p$ fusion reaction, $p p \rightarrow d e^{+} \nu_{e}$, is the primary solar nuclear reaction that essentially controls the burning rate of the sun, and hence the exact value of its cross section is a crucial input for any further developments of solar models. According to the latest review [4], this rate is required to be known with better than $\sim 1 \%$ precision in order to give impact to the existing models. Meanwhile, the Hep reaction, ${ }^{3} \mathrm{He}+p \rightarrow{ }^{4} \mathrm{He}+e^{+}+\nu_{e}$ is important in a different context. Although Hep plays only a very minor role in the pp-chain solar burning, it generates highestenergy solar neutrinos whose spectrum extends beyond the maximum energy of the ${ }^{8} \mathrm{~B}$ neutrinos. So, even though the flux of the Hep neutrinos is small, it can distort the higher end of the ${ }^{8} \mathrm{~B}$ neutrino spectrum [5], and this distortion can affect the interpretation of the results of a Super-Kamiokande experiment [6]. On the theoretical side, as explained in more detail below, Hep represents a unique case in which the leading-order contribution is drastically suppressed. This implies that Hep offers a good place for investigating higher order terms in EFT expansion. As for the $\nu$ - $d$ reactions, their primary importance is connected to the Sudbury Neutrino Observatory (SNO) experiments, in which the solar neutrino flux was monitored using a heavy water Cerenkov counter. As is well known, the SNO experiments [7] have established that the total solar neutrino flux (summed over all neutrino flavors) agrees with the prediction of the standard solar model [8], whereas the electron neutrino flux from the sun is significantly smaller than the total solar neutrino flux. These results of the SNO experiments have given "smoking-gun" evidence for the transmutation of solar electron neutrinos into neutrinos of other flavors. Obviously, a precise knowledge of the $\nu$ - $d$ reaction cross sections is of primary importance in interpreting the SNO data.

Before going into the description of achievements obtained in nuclear EFT, it seems useful to take a quick look at the latest lattice QCD calculation [9] of the nucleon weak form factors defined as

$$
\begin{aligned}
& <n\left|V^{\mu}\right| p>=\bar{u}_{n}\left[F_{V}\left(q^{2}\right) \gamma^{\mu}+\frac{i F_{M}\left(q^{2}\right)}{2 m_{N}} \sigma^{\mu \nu} q_{\nu}\right] u_{p}, \\
& <n\left|A^{\mu}\right| p>=\bar{u}_{n}\left[F_{A}\left(q^{2}\right) \gamma^{\mu} \gamma^{5}+\frac{F_{P}\left(q^{2}\right)}{m_{\mu}} q^{\mu} \gamma^{5}\right] u_{p},
\end{aligned}
$$

where $q=p_{n}-p_{p}$. The calculation [9] was carried out for the $u / d$ quark mass that corresponded to $m_{\pi}=0.33 \mathrm{GeV} \sim 0.67 \mathrm{GeV}$, and the form factors were calculated for $0.2 \mathrm{GeV}^{2}<q^{2}<0.75 \mathrm{GeV}^{2}$. Here is a partial summary of the results: (1) $F_{V}\left(q^{2}\right)$ is described by the conventional dipole form, but the dipole mass $M_{1}$ is found to be $27 \%$ smaller than the empirical value; $(2) F_{M}\left(q^{2}\right)$ is described by the dipole form, but the corresponding dipole mass $M_{2}$ is found to be $26 \%$ smaller than the empirical value; (3) $g_{A} / g_{V}=1.19$, which is $7 \%$ smaller than the experimental value $\left(g_{A} / g_{V}\right)_{\exp }=1.2695 \pm 0.0029$ (PDG08); (4) $F_{A}\left(q^{2}\right)$ is found to have the dipole form but the axial dipole mass $M_{A}$ is found to be $37 \%$ larger than $M_{A}^{\exp }$; (5) $\frac{\left(q^{2}-m_{\pi}^{2}\right) F_{P}\left(q^{2}\right)}{2 m_{N} F_{A}\left(q^{2}\right)} \equiv \alpha\left(q^{2}\right)$ is found to be constant in conformity with pion-pole dominance, but $\alpha=0.8 \sim 0.9$ (instead of $\alpha=1$ expected from PCAC). These features lead us to expect that it will be a while before nuclear elecroweak processes can be 
calculated with required precision by lattice QCD.

\section{Theoretical frameworks}

\section{- Standard nuclear physics approach (SNPA)}

The phenomenological potential picture has been highly successful in describing many different nuclear phenomena. In this picture an $A$-nucleon system is described by a Hamiltonian of the form

$$
H=\sum_{i}^{A} t_{i}+\sum_{i<j}^{A} V_{i j}+\sum_{i<j<k}^{A} V_{i j k}+\cdots,
$$

where $t_{i}$ is the kinetic energy of the $i$-th nucleon, $V_{i j}$ is a phenomenological two-body potential, $V_{i j k}$ is a phenomenological three-body potential, and so on. Once $H$ is specified, the $A$-body nuclear wave function $|\Psi\rangle$ is obtained by solving exactly or essentially exactly the Schrödinger equation

$$
H|\Psi>=E| \Psi>
$$

There is much freedom in choosing possible forms of $V_{i j}$ apart from a well-established requirement that, as the inter-nucleon distance $r_{i j}$ becomes sufficiently large, $V_{i j}$ should approach the one-pion exchange Yukawa potential. For the model-dependent short-range part of $V_{i j}$, one assumes certain functional forms and fix the parameters appearing therein by demanding that the solutions of eq.(3) for the $A=2$ case reproduce the nucleon-nucleon scattering data (typically up to the pion-production threshold energy) as well as the deuteron properties. There exist several so-called high-precision phenomenological N-N potentials that can reproduce all the existing two-nucleon data with normalized $\chi^{2}$ values close to 1 . These potentials differ significantly in the ways they parameterize short-range physics and, as a consequence, they exhibit substantial difference in their off-shell behavior. An important question therefore is to what extent the model-dependent short-range part affects the reliabilities of theoretical values of various observables in $A=2$ and higher systems calculated with these phenomenological potentials.

Nuclear responses to external electroweak probes in normal circumstances are given, to good approximation, by one-body terms, which are also called the impulse approximation (IA) terms. To achieve higher accuracy, however, we must also consider exchange current (EXC) terms, which represent the contributions of nuclear responses involving two or more nucleons. If for some reason the IA contributions are suppressed, it becomes particularly important to consider the EXC contributions. These exchange currents are usually taken to be two-body operators, and they are derived from one-boson exchange diagrams; the vertices featuring in the relevant diagrams are determined to satisfy the requirements of low-energy theorems and current algebra $[2,10]$. A formalism based on this picture is commonly called the standard nuclear physics approach (SNPA). (This is also called a potential model 
in the literature.) Schematically, an electroweak nuclear matrix element in SNPA is given by

$$
\mathcal{M}_{f i}^{S N P A}=<\Psi_{f}\left|\sum_{\ell}^{A} \mathcal{O}_{\ell}+\sum_{\ell<m}^{A} \mathcal{O}_{\ell m}+\sum_{\ell<m<n}^{A} \mathcal{O}_{\ell m n}\right| \Psi_{i}>,
$$

where the initial (final) nuclear wave function, $\Psi_{i}\left(\Psi_{f}\right)$, is a solution of eq.(3), and $\mathcal{O}_{\ell}, \mathcal{O}_{\ell m}$ and $\mathcal{O}_{\ell m n}$ are, respectively, one-body, two-body and three-body transition operators for a given electroweak process. SNPA has been used extensively for describing electroweak processes in light nuclei, and general good agreement found between theory and experiment [3] strongly suggests that SNPA captures much of the physics involved.

\section{- Effective field theory (EFT)}

Even though SNPA has been scoring great successes in describing a vast variety of data, it is still important from the fundamental point of view to raise the following issues. First, given the fact that hadrons and hadronic systems (such as nuclei) are governed by quantum chromodynamics (QCD), it is desirable to establish connections between SNPA with QCD. In this connection we note that, whereas chiral symmetry is known to be a fundamental symmetry of QCD, the formulation of SNPA is largely disjoint from this symmetry. Secondly, in introducing a phenomenological SNPA Lagrangian for describing the nuclear interaction and nuclear responses to the electroweak currents, it is not clear whether there is any identifiable expansion parameter that helps us to control the possible forms of terms in the Lagrangian and that gives a general measure of errors in our calculation.

An EFT-based approach [11] is a powerful tool to address these issues, and it has been studied with great intensity; for reviews, see [12]-[16]. The basic idea of EFT is simple. To describe phenomena characterized by a typical energy-momentum scale $Q$, we expect that we can exclude from our Lagrangian those degrees of freedom that belong to energy-momentum scales much higher than $Q$. So we introduce a cut-off scale $\Lambda$ that is sufficiently larger than $Q$ and we categorize our fields (to be generically represented by $\phi$ ) into two groups: high-frequency fields $\phi_{\mathrm{H}}$ and lowfrequency fields $\phi_{\mathrm{L}}$. By integrating out $\phi_{\mathrm{H}}$, we arrive at an effective Lagrangian that only contains $\phi_{\mathrm{L}}$ as explicit dynamical variables. The effective Lagrangian $\mathcal{L}_{\text {eff }}$ is related to the original Lagrangian $\mathcal{L}$ as

$$
\int[d \phi] \mathrm{e}^{\mathrm{i} \int d^{4} x \mathcal{L}[\phi]}=\int\left[d \phi_{\mathrm{H}}\right]\left[d \phi_{\mathrm{L}}\right] \mathrm{e}^{\mathrm{i} \int d^{4} x \mathcal{L}\left[\phi_{\mathrm{H}}, \phi_{\mathrm{L}}\right]} \equiv \int\left[d \phi_{\mathrm{L}}\right] \mathrm{e}^{\mathrm{i} \int d^{4} x \mathcal{L}_{\mathrm{eff}}\left[\phi_{\mathrm{L}}\right]} .
$$

$\mathcal{L}_{\text {eff }}$ defined by eq.(5) inherits the symmetries (and the pattern of symmetry breaking, if there is any) of the original Lagrangian $\mathcal{L}$. It can be shown that $\mathcal{L}_{\text {eff }}$ is given by the sum of all possible monomials of $\phi_{\mathrm{L}}$ and their derivatives that are consistent with the symmetry requirements dictated by $\mathcal{L}$. Because a term involving $n$ derivatives scales like $(Q / \Lambda)^{n}$, the terms in $\mathcal{L}_{\text {eff }}$ can be organized into a perturbative series wherein $Q / \Lambda$ serves as an expansion parameter. The coefficients of terms in this expansion are called low-energy constants (LECs). If all the LEC's up to a specified order $n$ can be fixed either from theory or from fitting to the experimental values of relevant 
observables, $\mathcal{L}_{\text {eff }}$ serves as a complete (and hence model-independent) Lagrangian to the given order of expansion. The results obtained are ensured to have accuracy of order $(Q / \Lambda)^{n+1}$. In applying EFT to nuclear systems, the underlying Lagrangian $\mathcal{L}$ is the QCD Lagrangian $\mathcal{L}_{Q C D}$, whereas, for the typical nuclear physics energymomentum scale $Q \ll \Lambda_{\chi} \sim 1 \mathrm{GeV}$, the effective degrees of freedom that should feature in $\mathcal{L}_{\text {eff }}$ are hadrons rather than the quarks and gluons. It is not trivial to apply the formal definition in eq.(5) to derive $\mathcal{L}_{\text {eff }}$ written in terms of hadrons starting from $\mathcal{L}_{Q C D}$; this is because the hadrons cannot be simply identified with the low-frequency field, $\phi_{L}$ in eq.(5). At present, the best one could do is to resort to symmetry considerations and the above-mentioned expansion scheme in the spirit of Weinberg's folk theorem [17]. Here chiral symmetry plays an important role. We know that chiral symmetry is spontaneously broken, generating the pions as Nambu-Goldstone bosons. ${ }^{2}$ This feature can be taken into account by assigning suitable chiral transformation properties to the Goldstone bosons and writing down all possible chiral-invariant terms up to a specified chiral order [18]. The above consideration presupposes exact chiral symmetry in $\mathcal{L}_{Q C D}$. In reality, however, $\mathcal{L}_{Q C D}$ contains small but finite quark mass terms, which explicitly break chiral symmetry and lead to a non-vanishing value of the pion mass $m_{\pi}$. Again, there is a welldefined method to determine what terms are needed in the Goldstone boson sector to represent the effect of explicit chiral symmetry breaking [18]. These considerations lead to an EFT called chiral perturbation theory $(\chi \mathrm{PT})[17,19]$. The successes of $\chi \mathrm{PT}$ in the meson sector are well documented; see, e.g., [12].

A serious problem we encounter in extending $\chi \mathrm{PT}$ to the nucleon sector is that, as the nucleon mass $m_{\mathrm{N}}$ is comparable to the cut-off scale $\Lambda_{\chi}$, a simple application of expansion in $Q / \Lambda$ does not work. This difficulty can be circumvented by employing heavy-baryon chiral perturbation theory $(\mathrm{HB} \chi \mathrm{PT})$, which essentially consists in shifting the reference point of the nucleon energy from 0 to $m_{\mathrm{N}}$ and in integrating out the small component of the nucleon field as well as the anti-nucleonic degrees of freedom. Thus an effective Lagrangian in $\mathrm{HB} \chi \mathrm{PT}$ contains as explicit degrees of freedom the pions and the large components of the redefined nucleon field. $\mathrm{HB} \chi \mathrm{PT}$ has as expansion parameters $Q / \Lambda_{\chi}, m_{\pi} / \Lambda_{\chi}$ and $Q / m_{\mathrm{N}}$. As $m_{\mathrm{N}} \approx \Lambda_{\chi}$, it is convenient to combine chiral and heavy-baryon expansions and introduce the chiral index $\bar{\nu}$ defined by $\bar{\nu}=d+(n / 2)-2$. Here $n$ is the number of fermion lines that participate in a given vertex, and $d$ is the number of derivatives (with $m_{\pi}$ counted as one derivative). A similar power counting scheme can be introduced for Feynman diagrams as well [11]. The contribution of a Feynman diagram that contains $N_{A}$ nucleons, $N_{E}$ external fields, $L$ loops and $N_{C}$ disjoint parts is shown to scale like $(Q / \Lambda)^{\nu}$, where the chiral index $\nu$ is defined as

$$
\nu=2 L+2\left(N_{C}-1\right)+2-\left(N_{A}+N_{E}\right)+\sum_{i} \bar{\nu}_{i}
$$

with the summation running over all the vertices contained in the Feynman diagram. $\mathrm{HB} \chi \mathrm{PT}$ has been applied with great success to the one-nucleon sector [12].

\footnotetext{
${ }^{2}$ We limit ourselves here to $\mathrm{SU}(2) \times \mathrm{SU}(2)$ chiral symmetry.
} 
However, $\mathrm{HB} \chi \mathrm{PT}$ cannot be applied in a straightforward manner to nuclei, which contain more than one nucleon. The reason is that nuclei involve very lowlying excited states, and the presence of this small energy scale spoils the original counting rule [11]. A method to avoid this difficulty was proposed by Weinberg [11]. Classify Feynman diagrams into two categories, irreducible and reducible diagrams. Irreducible diagrams are those in which every intermediate state has at least one meson in flight; all others are classified as reducible diagrams. One then applies the above chiral counting rules only to irreducible diagrams. The contributions of all the two-body irreducible diagrams (up to a specified chiral order) are treated as an effective potential (to be denoted by $V_{i j}^{E F T}$ ) acting on nuclear wave functions. Meanwhile, the contributions of reducible diagrams can be incorporated [11] by solving the Schrödinger equation

$$
H^{E F T}\left|\Psi>^{E F T}=E\right| \Psi>^{E F T},
$$

where

$$
H^{E F T}=\sum_{i}^{A} t_{i}+\sum_{i<j}^{A} V_{i j}^{E F T}+\sum_{i<j<k}^{A} V_{i j k}^{E F T}+\ldots,
$$

We may refer to this two-step procedure as nuclear $\chi \mathrm{PT}$ or, more specifically, nuclear $\chi \mathrm{PT}$ in the Weinberg scheme. ${ }^{3}$

To apply nuclear $\chi \mathrm{PT}$ to a nuclear electroweak process, we derive a nuclear transition operator $\mathcal{T}$ from a complete set of all the irreducible diagrams (up to a given chiral order $\nu$ ) involving the relevant external current(s) [20,21, 22]. Thus

$$
\mathcal{T}^{\mathrm{EFT}}=\sum_{i}^{A} \mathcal{O}_{i}^{\mathrm{EFT}}+\sum_{i<j}^{A} \mathcal{O}_{i j}^{\mathrm{EFT}}+\cdots
$$

To preserve consistency in chiral counting, the matrix element of $\mathcal{T}^{\mathrm{EFT}}$ must be calculated with the use of nuclear wave functions generated with the use of nuclear interactions that represent all the irreducible $A$-nucleon diagrams up to $\nu$-th order. Thus a transition matrix in nuclear EFT is given by

$$
\mathcal{M}_{f i}^{\mathrm{EFT}}=<\Psi_{f}^{\mathrm{EFT}}\left|\mathcal{T}^{\mathrm{EFT}}\right| \Psi_{i}^{\mathrm{EFT}}>=<\Psi_{f}^{\mathrm{EFT}}\left|\sum_{i}^{A} \mathcal{O}_{i}^{\mathrm{EFT}}+\sum_{i<j}^{A} \mathcal{O}_{i j}^{\mathrm{EFT}}+\cdots\right| \Psi_{i}^{\mathrm{EFT}}>,
$$

where the superscript, "EFT", means that the relevant quantities are calculated according to EFT as described above. If this program is carried out rigorously, it would constitute an ab initio calculation. Let us call it "AB-EFT". It is to be noted that in EFT we know exactly at what chiral order three-body operators start to contribute to $\mathcal{T}$, and that, to chiral orders relevant to most applications described below, there is no need for three-body operators. With this understanding, we have kept only one- and two-body operators in eq.(10). The unambiguous classification of transition operators according to their chiral orders is a great advantage of EFT, which is missing in eq.(4).

\footnotetext{
${ }^{3}$ This is often called the $\Lambda$-counting scheme [14].
} 
We should point out that there exists an alternative form of nuclear EFT called the PDS scheme, proposed by Kaplan, Savage and Wise [23], which uses a counting scheme (often called Q-counting) different from the Weinberg scheme. Although many important results have been obtained in the PDS scheme (for a review, see e.g. [15]), we concentrate here primarily on the Weinberg scheme.

We also remark that, for very low energy nuclear phenomena whose typical energy-momentum scale is $Q \ll m_{\pi}$, even the pions can be treated as "heavy" particles and can be eliminated from $\mathcal{L}_{\text {eff }}$, with the choice of $\Lambda \approx m_{\pi}$. This leads to pionless $\operatorname{EFT}[23,24,25]$. Here there is no manifest chiral symmetry since there is no explicit pion. In this regime the $N N$ interactions and electroweak currents are described by point-like contact terms.

\section{- Hybrid EFT}

In the above we emphasized the formal merits of nuclear EFT. In actual calculations, however, we need to consider the following two points. First, it is still a big challenge to generate, strictly within the EFT framework, nuclear wave functions the accuracy of which is comparable to that of SNPA wave functions. Secondly, whereas a chiral Lagrangian, $\mathcal{L}_{\text {eff }}$, is definite only when the values of all the relevant LECs are fixed, this condition cannot be readily satisfied in many cases. A pragmatic solution to the first difficulty [26]-[31] is to use in eq.(10) wave functions obtained in SNPA; this eclectic approach is called hybrid EFT. A nuclear transition matrix element in hybrid EFT is given by

$$
\mathcal{M}_{f i}^{h y b-E F T}=<\Psi_{f}^{S N P A}\left|\sum_{\ell}^{A} \mathcal{O}_{\ell}^{E F T}+\sum_{\ell<m}^{A} \mathcal{O}_{\ell m}^{E F T}\right| \Psi_{i}^{S N P A}>,
$$

Because, as mentioned, the NN interactions that generate SNPA wave functions reproduce accurately the two-nucleon data, the adoption of eq.(11) is almost equivalent to using the empirical data themselves to control the initial and final nuclear wave functions. From a purely formal point of view, hybrid EFT may be deemed as a "regression". But, if our goal is to obtain a transition matrix element as accurately as possible with the maximum help of available empirical input, hybrid EFT does have a legitimate status. It offers more flexibility and predictive power than the AB-EFT, and it can be highly accurate so long as the off-shell problem and the contributions of three-body (and higher-body) terms are properly addressed. The issue of possible unknown LECs will be discussed in connection with MEEFT in the next subsection.

\section{- MEEFT}

A great merit of hybrid EFT is that it can be used for complex nuclei $(A=3$, $4, \ldots)$ with essentially the same accuracy and ease as for the $A=2$ system. ${ }^{4}$ We

\footnotetext{
${ }^{4}$ Here we are ignoring "purely technical" complications that can grow in actual numerical calculations for higher- $A$ systems.
} 
reemphasize in this connection that the contributions of transition operators involving three or more nucleons, which can in principle contribute in $A$-nucleon systems $(A \geq 3)$, are intrinsically suppressed according to chiral counting, and hence, up to a certain chiral order, a transition operator in an $A$-nucleon system consists of the same EFT-based 1-body and 2-body terms as used for the two-nucleon system. Then, since SNPA provides high-quality wave functions for the $A$-nucleon system, one can calculate $\mathcal{M}_{f i}^{h y b-E F T}$ with precision comparable to that for the corresponding two-nucleon case.

In most cases the one-body operator, $\mathcal{O}_{\ell}^{E F T}$, is free from unknown LECs. So we may concentrate on the two-body operator, $\mathcal{O}_{\ell m}^{E F T}$. Suppose that $\mathcal{O}_{\ell m}^{E F T}$ under consideration contains an LEC (call it $\kappa$ ) that cannot be determined with the use of $A=2$ data alone. It can happen that an observable (call it $\Omega$ ) in a A-body system $(A \geq 3)$ is sensitive to $\kappa$ and that the experimental value of $\Omega$ is known with sufficient accuracy. We then can determine $\kappa$ by calculating $\mathcal{M}_{f i}^{\text {hyb-EFT }}$ responsible for $\Omega$ and adjusting $\kappa$ to reproduce the experimental value of $\Omega$. Once $\kappa$ is determined this way, we can make predictions for any other observables for any other nuclear systems that are controlled by the same transition operators. When hybrid EFT is used in this manner, it is called more effective effective field theory (MEEFT) [30, 31]. ${ }^{5}$

MEEFT is a powerful formalism for correlating various observables in different nuclei, using the transition operators controlled by EFT. A further practical advantage of MEEFT is that, since correlating the observables in neighboring nuclei is expected to serve as an additional renormalization, the possible effects of higher chiral order terms and/or off-shell ambiguities can be significantly suppressed with the use of MEEFT. We will come back to this later, when we discuss concrete examples.

\section{Examples}

\section{- $\quad p p$-fusion, $\nu d$ reactions and $\mu d$ capture}

A common feature of these reactions is that a precise knowledge of the GamowTeller (GT) transition matrix elements is required in estimating their cross sections. We show here, following Refs. [31], that the idea of MEEFT can be used very neatly for this group of reactions. The 1-body IA operators for the GT transition can be fixed unambiguously from the available 1-body data. As for the 2-body operators, to next-to-next-to-next-to-leading order $\left(\mathrm{N}^{3} \mathrm{LO}\right)$ in chiral counting, there appears one unknown LEC that at present cannot be determined from data for the $A=2$ systems. This unknown LEC, denoted by $\widehat{d}_{R}$ in [27], parametrizes the strength of contact-type four-nucleon coupling to the axial current. Park et al. [31] noticed that the same LEC, $\widehat{d}_{R}$, also features as the only unknown parameter in the calculation of the tritium $\beta$-decay rate $\Gamma_{\beta}^{t}$, and they proposed to use MEEFT to place a constraint

\footnotetext{
${ }^{5}$ It is also called EFT*. MEEFT should be distinguished from an earlier naive hybrid EFT model wherein the short-range terms were dropped altogether using an intuitive argument based on short-range NN repulsion.
} 
on $\widehat{d}_{R}$ from the experimental information on $\Gamma_{\beta}^{t}$. Since the empirical value of $\Gamma_{\beta}^{t}$ is known with high precision, and since the accurate wave functions of ${ }^{3} \mathrm{H}$ and ${ }^{3} \mathrm{He}$ are available from SNPA [32], we can determine $\widehat{d}_{R}$ with sufficient accuracy for our purposes. Once the value of $\widehat{d}_{R}$ is determined this way, we can carry out parameterfree MEEFT calculations for $p p$-fusion [31], $\nu$ - $d$ reactions [33], and $\mu$ - $d$ capture. (An on-going attempt to deduce $\widehat{d}_{R}$ from $\mu$ - $d$ capture will be discussed later.)

We should mention the important role of momentum cutoff in MEEFT. As emphasized before, the effective Lagrangian $\mathcal{L}_{\text {eff }}$ is, by construction, valid only below the specified cutoff scale $\Lambda$. Needless to say, this basic constraint should be respected in our nuclear EFT calculations, and for that we must make sure that nuclear intermediate states involved in the computation of eq.(10) do not get out of this constrained world. Since, however, the use of $V^{\text {phenom }}$ introduces high momentum components above $\Lambda_{\mathrm{QCD}}$, we need to introduce momentum cutoff at $\Lambda_{N N}$ to eliminate high-momentum components. It is reasonable to implement this constraint by requiring that the two-nucleon relative momentum should be smaller than $\Lambda_{N N}$. Park et al. [31] used a Gaussian cutoff function proportional to $\exp \left(-\vec{p}^{2} / \Lambda_{N N}^{2}\right)$ but its detailed form should not be too important. As a reasonable range of the value of $\Lambda_{N N}$ we may choose: $500 \mathrm{MeV} \lesssim \Lambda_{N N} \lesssim 800 \mathrm{MeV}$, where the lower bound is dictated by the requirement that $\Lambda_{N N}$ should be sufficiently larger than the pion mass (to fully accommodate pion physics), while the upper bound reflects the fact that our EFT is devoid of the $\rho$ meson. Mismatch between $\Psi^{\mathrm{EFT}}$ and $\Psi^{\mathrm{SNPA}}$ only affects short-distance behavior, and we expect that $\Lambda_{N N^{-}}$-independence is a measure of model independence of an MEEFT calculation.

For a given value of $\Lambda_{N N}$ within the above range, $\widehat{d}_{R}$ is tuned to reproduce $\Gamma_{\beta}^{t}$, and then the rates for pp-fusion, the $\nu d$ reactions and $\mu d$ capture are calculated. Before giving an account of the individual results, we should point out a notable common feature. Although the optimal value of $\widehat{d}_{R}$ varies significantly as a function of $\Lambda_{N N}$, the observables (in our case the above three reaction rates) exhibit remarkable stability against the variation of $\Lambda_{N N}$ (within the above-discussed physically reasonable range). This stability may be taken as an indication that the use of MEEFT for inter-correlating the observables in neighboring nuclei effectively renormalizes various effects, such as the contributions of higher-chiral order terms, mismatch between the SNPA and EFT wave functions, etc. This stability is essential in order for MEEFT to maintain its predictive power.

\subsection{The $p p$-fusion S-factor}

The $S$-factor is related to the cross section $\sigma$ as

$$
\sigma(E)=\frac{S(E)}{E} \exp \{-2 \pi \eta(E)\}
$$

where $E$ is the incident energy in the center-of-mass system, and $\eta(E) \equiv \frac{Z_{1} Z_{2} e^{2}}{\hbar v}$ is the Coulomb penetration factor. The $S$-factor for $p p$-fusion at threshold is related 
to the transition matrix element $\mathcal{M}$ as [4]

$$
S_{p p}(0)=6 \pi^{2} m_{p} \alpha \ln 2 \frac{1}{\gamma^{3}}\left(g_{A} / g_{V}\right)^{2} \frac{f_{p p}^{R}}{(f t)_{0^{+} \rightarrow 0^{+}}}|\mathcal{M}|^{2}
$$

where $\gamma=\left(2 \mu B_{d}\right)^{1 / 2}=0.2316 \mathrm{fm}^{-1}$ is the deuteron binding wave number, and $f_{p p}^{R}$ is the phase space factor including radiative corrections. As mentioned, the solar model and stellar evolution theory require $1 \%$ precision in $S_{p p}$ [4].

It is useful to first recall what was achieved in SNPA. A benchmark calculation of $S_{p p}$ in SNPA was carried out by Schiavilla et al. [34]. These authors took full advantage of the fact, first pointed out in Ref. [35], that the Gamow-Teller (GT) matrix elements for $p p$-fusion and tritium $\beta$-decay are closely correlated. For each of the two-body GT transition operators with different $r$-dependences $(r=$ relative distance of the two nucleons), the transition density for $p p$-fusion is found to be accurately proportional to that for tritium $\beta$-decay. This means that a single multiplicative constant can match them all. Schiavilla et al. [34] fine-tuned the $N \Delta$-axial coupling constant, which featured in a dominant two-body axial current diagram $(\Delta$-particle excitation diagram), and which was only loosely controlled from other sources of information. After this fine-tuning, $S_{p p}$ was calculated with estimated uncertainties of $\sim 0.3 \%$; here the error estimate came from the range of variation in $S_{p p}(0)$ for the five different high-precision phenomenological potentials used in [34]. A further examination of this error estimation will be described below.

Park et al. [31] carried out an MEEFT calculation of $S_{p p}(0)$ and obtained

$$
S_{p p}(0)=3.94 \times(1 \pm 0.008) \times 10^{-25} \mathrm{MeV} \mathrm{b} .
$$

$S_{p p}(0)$ is found to change only by $\sim 0.1 \%$ against changes in $\Lambda_{N N}$, ensuring the robustness of the MEEFT calculation in this respect. The convergence pattern in chiral expansion for the $p p$-fusion transition amplitude is reported to be [31]: $\mathcal{M} \sim(1+0+0.1 \%+0.9 \%+0.4 \%)$. It is noted that the 2 nd and 3rd order terms are accidentally small, but that the 4 th and 5 th order are roughly in conformity with expansion in $m_{\pi} / \Lambda_{\chi} \approx \frac{1}{7}$. The 3-body currents contribute at the sixth order. Uncertainties due to the higher order contributions estimated from the size of the 5th-order contribution are $0.4 \%$ (in amplitude), or $0.8 \%$ (in probability). ${ }^{6}$ The MEEFT result, eq.(13), is consistent with that obtained in SNPA [34]. Meanwhile, the fact that MEEFT allows us to make error estimation is a notable advantage over SNPA. It is worth emphasizing that the accuracy achieved in eq.(13) represents an improvement by a factor of $\sim 5$ over the previous results based on a naive hybrid EFT [27].

The calculation of $S_{p p}$ in pionless EFT was performed up to NNLO by Kong and Ravndal [36], and up to fifth order by Butler and Chen [37]; see also Ando

\footnotetext{
${ }^{6}$ In [31], the relative error of $0.4 \%$ was assigned to $S_{p p}(0)$. However, it has been found subsequently that the $0.4 \%$ uncertainty should have been assigned to the transition amplitude, not to the transition probability; we therefore double the error estimate quoted in [31] and adopt 0.008 in eq. (13).
} 
et al. [38]. Here again, up to the order considered, there is only one unknown LEC, denoted by $L_{1, A}$, which (analogously to $\widehat{d}^{R}$ ) parameterizes the strength of four-nucleon contact axial-vector coupling. The results of Refs. [36, 37] are: (i) the central value of $S_{p p}(0)$ is consistent with those obtained in SNPA and MEEFT; (ii) the theoretical uncertainty in $S_{p p}(0)$ is $\sim 3 \%$, which is significantly larger than the results obtained in SNPA and MEEFT. One thing to be noted here is that the PDS scheme [23] (used in pionless EFT) adopts an expansion scheme for transition amplitudes themselves, without employing the concepts of the potential or wave functions. In certain contexts this feature may be an advantage, but its disadvantage in the present context is that one cannot readily relate the transition matrix elements for an $A$-nucleon system with those for the neighboring nuclei; in PDS, each nuclear system requires a separate parameterization. Therefore, $L_{1 A}$ in pionless EFT needs to be determined from the empirical value of a 2-body observable, which however is at present available only with large experimental error. This situation is reflected in the rather large $3 \%$ uncertainty in $S_{p p}(0)$ calculated in pionless EFT.

In a very recent review [4], there has been an attempt to deduce the most updated estimate for $S_{p p}(0)$, and the recommended value is

$$
S_{p p}(0)=4.01 \times(1 \pm 0.009) \times 10^{-24} \mathrm{MeV} \text { b. }
$$

This estimate arises as follows. In eq.(12), the matrix element $\mathcal{M}$ is assigned the value corresponding to the central value obtained in SNPA [34] and MEEFT [31]; these two approaches give exactly the same value. For the other inputs that enter into eq.(12), the most updated values are used; some of them slightly differ from the values used in the earlier publications. The relative uncertainty of 0.009 in eq.(14) is dominated by the $0.8 \%$ uncertainty in $|\mathcal{M}|^{2}$ estimated from the MEEFT result [31] and the $0.5 \%$ uncertainty coming from the experimental error in the $n n$ scattering length $a_{n n}$. These uncertainties and other smaller uncertainties are added in quadrature. Eq.(14) indicates that $S_{p p}(0)$ now can be estimated within $1 \%$ precision.

\section{$3.2 \nu$ - $d$ reactions}

As mentioned, $\nu$ - $d$ reactions are important in connection with the SNO experiments on neutrino oscillations. Within SNPA a detailed calculation of the $\nu$ - $d$ cross sections, $\sigma(\nu d)$, was carried out in Refs. [39, 40, 41]. Nakamura et al. [41], following Schiavilla et al.'s approach [34], used the two-body current whose strength was fine-tuned to reproduce the tritium $\beta$-decay rate. With the use of this fine-tuned two-body exchange current, Nakamura et al. calculated $\sigma(\nu d)$ for four types of reactions: charged-current and neutral-current reactions on $d$ for both neutrino and anti-neutrino cases. Meanwhile, Butler, Chen and Kong (BCK) [42] carried out a pionless-EFT calculation of the $\nu$ - $d$ cross sections. The results obtained agree with those of Nakamura et al. [41] in the following sense. A pionless-EFT calculation, as mentioned in the discussion of $p p$-fusion, involves one unknown LEC, $L_{1 \mathrm{~A}}$, which 
represents the strength of a four-nucleon axial-current coupling term. BCK determined $L_{1 \mathrm{~A}}$ by requiring that the $\nu d$ cross sections of NETAL be reproduced by their pionless-EFT calculation. With the value of $L_{1 \mathrm{~A}}$ fine-tuned this way, the $\sigma(\nu d)^{\text {'s }}$ obtained by BCK show a perfect agreement with those of Nakamura et al. for all the four reactions mentioned above, and for the entire solar neutrino energy range, $E_{\nu} \lesssim 20 \mathrm{MeV}$. Moreover, the optimal value, $L_{1 \mathrm{~A}}=5.6 \mathrm{fm}^{3}$, found by BCK [42] is consistent with the order of magnitude of $L_{1 \mathrm{~A}}$ expected from the naturalness argument (based on a dimensional analysis), $\left|L_{1 \mathrm{~A}}\right| \leq 6 \mathrm{fm}^{3}$. The fact that an EFT calculation (with one parameter fine-tuned) reproduces the results of SNPA very well strongly suggests the robustness of the SNPA results for $\sigma(\nu d)$.

Even though it is reassuring that the $\nu$ - $d$ cross sections calculated in SNPA and pionless-EFT agree with each other (in the sense explained above), it is desirable to carry out an EFT calculation that is free from any adjustable LEC. Fortunately, MEEFT allows us to carry out an EFT-controlled parameter-free calculation of the $\nu$ - $d$ cross sections, because the only LEC appearing therein, $\widehat{d}_{R}$, has already been determined from $\Gamma_{\beta}^{t}[31]$. Ando et al. [33] took advantage of this situation and carried out an MEEFT calculation of $\sigma(\nu d)$. The results were found to agree within $1 \%$ with $\sigma(\nu d)$ 's obtained in SNPA [41]. These results show that the $\nu$ - $d$ cross sections used in interpreting the SNO experiments [7] are reliable at the $1 \%$ precision level.

There have been subsequent attempts to calibrate $L_{1 A}$ from reactor $\bar{\nu}$-deuteron breakup reactions [43], and also from a "self-calibrating" analysis of the SNO data [44]. The $\sigma(\nu d)$ 's corresponding to the range of $L_{1 A}$ obtained in these analyses are consistent with the $\sigma(\nu d)$ 's obtained in SNPA and MEEFT. However, the resulting constraints on $L_{1 A}$ are not stringent. We have mentioned that both $L_{1 A}$ and $\widehat{d}_{R}$ represent the strength of axial-current-four-nucleon contact coupling. It is to be noted, however, that $L_{1 A}$ belongs to pion-less EFT, while $\widehat{d}_{R}$ to pion-ful EFT. In the pion-ful EFT, because of the strong tensor force, the exchange current involving the deuteron $d$-state is important, and the s-wave exchange current arising from the $\widehat{d}_{R}$ term is separate from this tensor-force effect. By contrast, in the pion-less EFT, the explicit $d$-wave term is a higher-order correction, and hence the $s$-wave $L_{1 A}$ term must subsume the strong tensor-force contributions. It would be informative to investigate the relation between $L_{1 A}$ and $\widehat{d}_{R}$ from this perspective.

\section{- Off-shell effects}

In introducing hybrid EFT, we have replaced $|\Psi\rangle^{E F T}$ for the initial and final nuclear states in eq.(10) with the corresponding $\mid \Psi>^{S N P A}$; see eq.(11). This replacement may bring in a certain degree of model dependence, called the off-shell effect, because the phenomenological NN interactions are constrained only by the on-shell two-nucleon observables. ${ }^{7}$ This off-shell effect, however, is expected to be small for the reactions under consideration, since they involve low momentum transfers and

\footnotetext{
${ }^{7}$ In a consistent theory, physical observables are independent of field transformations that lead to different off-shell behaviors, and therefore the so-called off-shell effect is not really a physical effect. In an approximate theory, observables may exhibit superficial dependence on off-shell behavior, and it is customary to refer to this dependence as an off-shell effect.
} 
hence are not extremely sensitive to the short-range behavior of the nuclear wave functions. One way to quantify this expectation is to compare a two-nucleon relative wave function generated by the phenomenological potential with that generated by an EFT-motivated potential. Phillips and Cohen [45] made such a comparison in their analysis of the 1-body operators responsible for electron-deuteron Compton scattering, and showed that a hybrid EFT should work well up to momentum transfer $700 \mathrm{MeV}$. A similar conclusion is expected to hold for a two-body operator, so long as its radial behavior is duly "smeared-out" reflecting a finite momentum cutoff. Thus, hybrid EFT as applied to low energy phenomena is expected to be practically free from the off-shell ambiguities. The off-shell effect should be even less significant in MEEFT, wherein an additional "effective" renormalization is likely to be at work (see subsection 3.1).

We now discuss briefly another interesting development, due to Tom Kuo and his colleagues [46], which can shed much light on the reliability of a hybrid EFT or MEEFT calculation. As mentioned, a "realistic phenomenological" nuclear interaction, $V_{i j}$ in eq.(2), is determined by solving the Schrödinger equation, eq.(3), for the $A=2$ system and fitting the results to the full set of two-nucleon data up to the pion production threshold energy. So, physically, $V_{i j}$ should reside in a momentum regime below a certain cutoff, $\Lambda_{c}$. In the conventional treatment, however, the existence of this cutoff scale is ignored, and eq.(3) is solved, allowing the entire momentum range to participate. Kuo et al. proposed to construct an effective low-momentum potential $V_{\text {low-k }}$ by eliminating (or integrating out) from $V_{i j}$ the momentum components higher than $\Lambda_{c}$, and calculated $V_{\text {low-k }}$ 's corresponding to many well-established examples of $V_{i j}$ 's. Remarkably, it was found that all these $V_{\text {low-k }}$ 's give identical results for the half-off-shell T-matrices, even though the ways short-range physics is encoded in these $V_{i j}$ 's are highly diverse. This implies that the $V_{\text {low-k }}$ 's are free from the off-shell ambiguities, and therefore the use of $V_{\text {low-k }}$ 's is essentially equivalent to employing $V_{i j}^{E F T}$ (that appeared in eq.(8)), which by construction should be model-independent. Now, as mentioned, our MEEFT calculation has a momentum-cutoff regulator, and this essentially ensures that the matrix element, $\mathcal{M}_{f i}^{h y b-E F T}$, in eq.(11) is only sensitive to the half-off-shell T-matrices that are controlled by $V_{\text {low }-k}$ instead of $V_{i j}$. Therefore, we can expect that the MEEFT results reported here are essentially free from the off-shell ambiguities.

Recently, Mosconi et al. [47] have compared $\nu d$ cross sections calculated for various models that differ in the way the "nuclear PCAC requirement" is implemented, and concluded from this comparison that the $\sigma(\nu d)$ 's obtained in SNPA, MEEFT and pionless EFT have uncertainties as large as 2-3\%. Mosconi et al. have reached this conclusion by examining the scatter of unconstrained calculations of $\sigma(\nu d)$. However, all state-of-the-art calculations use $\Gamma_{\beta}^{t}$ to reduce two-body current and other uncertainties. Once this constraint is imposed, the scatter in the calculated values of $\sigma(\nu d)$ should be significantly reduced. 


\section{$3.3 \mu d$ capture and MuSun experiment}

Although it is likely that the determination of $\widehat{d}_{R}$ from $\Gamma_{\beta}^{t}$ is good enough for practical purposes, it is worthwhile to study a possibility to fix $\widehat{d}_{R}$ with the use of an observable belonging to the $A=2$ systems. A promising candidate is the $\mu d$-capture process, $\mu^{-}+d \rightarrow \nu_{\mu}+n+n$. Even though rather large energy-momentum transfers caused by the disappearance of a $\mu^{-}$seem to make the applicability of EFT here a delicate issue, we can show [48] that, as far as the hadron sector is concerned, $\mu$ - $d$ capture is in fact a reasonably "gentle" process. This is because: (1) the $\nu_{\mu}$ carries away most of the energy, and (2) there is a large enhancement of the transition amplitude in a kinematic region where the relative motion of the final two nucleons is low enough to justify the use of EFT. Thus $\mu d$ capture can be used for controlling $\widehat{d}_{R}$, if the quality of experimental data improves sufficiently. The MuSun experiment at PSI (proposed in 2008) [49] aims to determine the $\mu d$ capture rate $\Gamma_{\mu d}$ with $\sim 1.5 \%$ accuracy; for a review, see [50]. This will enable us to determine $\widehat{d}^{R}$ within $A=2$ system. In this connection, it is important to point out that $g_{P} \equiv F_{A}\left(q^{2}=-0.88 m_{\mu}^{2}\right)$ is totally under control by now. Previously, the main goal of $\mu$-nucleus capture experiments was to obtain information on $g_{P}$ given the fact that $\mu$ capture goes much faster on nuclei (with high $Z$ ) than on a proton. This goal, however, has been perennially elusive because of nuclear physics complications. It is therefore highly noteworthy that a $\mathrm{HB} \chi \mathrm{PT}$ calculation [51] gives a robust prediction, $g_{P}=8.26 \pm 0.23$, and that this value is in good agreement with the experimental value, $g_{P}=7.7 \pm 1.1$, obtained in the MuCap Experiment at PSI [52]. The fact that the 1-body current is well known allows us to concentrate on the 2-body part.

Recent SNPA calculations of $\mu$ - $d$ capture can be found in Refs. [53, 54, 55]; According to Tatara et al. [53], the hyperfine-doublet capture rate $\Gamma_{d}$ (by far the dominant capture channel) is $\Gamma_{d}=397-400 \mathrm{~s}^{-1}$, and the results are stable against the use of different $N N$ potentials available at the time of their work. The latest calculation by Ricci et al. [55] reports $\Gamma_{d}=416 \mathrm{~s}^{-1}$, and that there is nonnegligible $N N$-potential dependence between the earlier and new-generation realistic phenomenological potentials. Thus the results obtained within SNPA exhibit some scatter, and although Ricci et al. offer rather detailed discussion of its possible origin, further investigation seems needed. An MEEFT calculation of $\Gamma_{d}$ was carried out by Ando et al. [48], yielding the value $\Gamma_{d}=386 \mathrm{~s}^{-1}$ with a high degree of stability against variations in $\Lambda_{N N}$. A pionless EFT calculation was performed by Chen et al. [56] but this approach is reliable only for limited kinematical regions, because of the very low momentum cutoff inherent with pionless EFT.

Comparison with experiment is complicated by the existence of two barely overlapping experimental results: $\Gamma_{d}^{\exp }=470 \pm 29 \mathrm{~s}^{-1}$ [57], and $\Gamma_{d}^{\exp }=409 \pm$ $40 \mathrm{~s}^{-1}$ [58]. All the above-mentioned theoretical values are consistent with the value of $\Gamma_{d}^{\exp }$ reported in [58], and disagree with that given in [57].

Since the MuSun experiment envisages to measure $\Gamma_{d}$ with $1.5 \%$ precision, it is important to match the accuracy of theoretical prediction. Most recently, a highly 
elaborate calculation of $\Gamma_{d}$ has been performed in both SNPA and MEEFT by Marcucci et al. $[59]^{8}$. The nuclear wave functions are generated from the Argonne $V_{18}$ potential (for SNPA) or from a chiral N3LO two-nucleon potential [60] (for MEEFT). As in the pp-fusion calculation, one parameter needs to be fixed (the $N$-to- $\Delta$ axial coupling constant in SNPA or $\widehat{d}_{R}$ in MEEFT). This is fixed by reproducing the tritium $\beta$-decay rate. The model dependence with respect to the adopted interactions and current (including cutoff dependence in the case of MEEFT) is found to be weak. Marcucci et al. give

$$
\Gamma_{d}=392 \pm 2.3 \mathrm{~s}^{-1}
$$

It is interesting to note that this result is close to that obtained by Ando et al. [48]. We also remark that what Marcucci et al. refer to as MEEFT is actually "less hybrid" in nature than the MEEFT used by Ando et al. in that the former employs the chiral N3LO two-nucleon potential instead of a phenomenological $N N$ potential. When the MuSun result becomes available, one can use it in two ways - compare it with the theoretical prediction in eq.(15) based on $\Gamma_{\beta}^{t}$, or use it to determine $\widehat{d}_{R}$ from the $A=2$ system (without referring to the $A=3$ system).

\subsection{Hep and Hen reactions}

\section{- Hep reaction}

As mentioned, the Hep reaction, ${ }^{3} \mathrm{He}+p \rightarrow{ }^{4} \mathrm{He}+\nu_{e}+e^{+}$, produces solar neutrinos of the highest maximum energy $\left(E_{\nu}^{\max }=18.8 \mathrm{MeV}\right)$, although the flux is extremely low. An accurate estimation of this cross section is a particularly challenging task because: (1) the contribution of the leading-order 1-body GT operator is highly suppressed due to the approximate wave function orthogonality [61], and (2) there is a strong cancellation between the 1-body and 2-body GT matrix elements [35, 32]. Reflecting this situation, estimates of $S_{\mathrm{Hep}}(0)$ changed by orders of magnitude from Salpeter's original value, $S_{\text {Hер }}(0)_{\text {Salpeter }}=630 \times 10^{-20} \mathrm{keV}$-b to modern values; for a review, see [62]. Park et al. [31] carried out an MEEFT calculation of $S_{\text {Hep }}(0)$ with the use of $\widehat{d}_{R}$ determined from $\Gamma_{\beta}^{t}$ (the same procedure as used in calculating $\left.S_{p p}(0)\right)$, and obtained

$$
S_{H e p}(0)=(8.6 \pm 1.3) \times 10^{-20} \mathrm{keV} \mathrm{b},
$$

where the error spans the range of the $\Lambda_{N N}$ dependence for $\Lambda_{N N}=500-800 \mathrm{MeV}$. Again, the MEEFT result agrees with that obtained in SNPA by Marcucci et al. [32]: $S_{\text {Hep }}(0)_{\mathrm{SNPA}}=9.6 \times 10^{-20} \mathrm{keV}$-b. The above-mentioned large cancellation between the 1-body and 2-body contributions in this case amplifies the cutoff dependence of $S_{\text {Hep }}(0)$, but the error quoted in eq.(16) is still small enough for the purpose of analyzing the existing Super-Kamiokande data [6].

\footnotetext{
${ }^{8}$ The authors of this work use the term EFT* instead of MEEFT.
} 


\section{- Hen reaction}

The Hen reaction, ${ }^{3} \mathrm{He}+n \rightarrow{ }^{4} \mathrm{He}+\gamma$, is very similar to Hep except, of course, the weak interaction is replaced by the EM interaction. Thus, just like the GamowTeller matrix element for Hep, the leading-order 1-body M1 matrix element for Hen is highly suppressed due to the pseudo-orthogonality of the initial and final nuclear wave functions. Moreover, there is strong cancellation between the hindered 1-body contribution and the 2-body exchange-current contribution, leading to a further suppression of the Hen reaction rate. Thus, like Hep, Hen is sensitive to higher order terms in chiral expansion. Meanwhile, the experimental value of $\sigma_{\text {Hen }}$ is known rather accurately: $\sigma_{\text {Hen }}^{\exp }=(54 \pm 6) \mu b[63]$, or $\sigma_{\text {Hen }}^{\exp }=(55 \pm 3) \mu b$ [64]. Therefore, Hen provides a good testing ground for the validity of MEEFT as used for Hep. A very elaborate MEEFT calculation of Hen was recently carried out by Lazauskas, Song and Park [65]. Up to $\mathrm{N}^{3} \mathrm{LO}$ considered by these authors, the M1 operators contain two LECs, which can be determined from the magnetic moments, $\mu\left({ }^{3} \mathrm{H}\right)$ and $\mu\left({ }^{3} \mathrm{He}\right)$. The initial and final nuclear wave functions are obtained from the rigorous Faddev-Yakubowski equations [66] for five sets of high-precision phenomenological potentials. Lazaukas et al.'s calculation gives

$$
\sigma_{\text {Hen }}=(38 \sim 58) \mu b,
$$

with high stability against changes in $\Lambda_{N N}$ in the range $500 \sim 900 \mathrm{MeV}$. The authors emphasized the importance of long-range nuclear correlation effects in obtaining an accurate estimate of $\sigma_{\text {Hen }}$. To explain this point, it is useful to first discuss what Song et al. [67] pointed out in their MEEFT study of the M1 properties of the $A=3$ nuclei. They noticed that the M1 matrix elements (MEs) in the $A=3$ nuclei calculated in MEEFT for various realistic potentials have strong correlation with the triton binding energies $B_{3}$ calculated with the corresponding realistic potentials. In fact, these quantities fall on a well-defined single curve. Meanwhile, since $B_{3}$ governs the long distance contribution to the MEs, the model dependence (that is, variations in MEs calculated for nuclear potentials that give different values for $B_{3}$ ) cannot be lifted by renormalizing the local (or short-range) operators. However, the use of the empirically found correlation curve between the MEs and $B_{3}$ allows us to drastically reduce scatters in the calculated MEs. This is achieved by introducing the constraint that one should accept only those values of the MEs which, along the correlation curve, have values of $B_{3}$ consistent with its experimental value. This constraint is found to essentially eliminate the model dependence in the MEs [67]. Now, Lazauskas et al. [65] noticed that a very similar feature exists also for Hen. Defining the quantity $\zeta$ by $\zeta \equiv\left[q\left(a_{n H e^{3}} / r_{H e^{4}}^{2}\right]^{-2.75}\right.$ ( $q=$ photon momentum), Lazauskas et al. found that $\sigma_{\mathrm{Hen}}$ and $\zeta$ are so well correlated that the $\sigma_{\mathrm{Hen}} \zeta$ plot forms a sharply defined straight line. Then, again, by accepting only those values of $\sigma_{\mathrm{Hen}}$ which are consistent with the empirical value of $\zeta$, one can deduce the model dependence of $\sigma_{\text {Hen }}$ significantly. The estimate of $\sigma_{\text {Hen }}$ given in eq.(17) was obtained through this method. ${ }^{9}$ The fact that this theoretical value is in good agreement with the above quoted experimental value renders strong support for the basic soundness of the

\footnotetext{
${ }^{9}$ The main point here is not a linear relation between $\sigma_{\text {Hen }}$ and $\zeta$; after all, the latter is
} 
MEEFT approach. We consider it noteworthy that MEEFT works well also for a highly suppressed process wherein higher-chiral order terms become important; see Ref. [68]. The examples of Hen and the MEs in the $A=3$ systems also demonstrate that it is profitable to avail ourselves of the merit of EFT (to control short-range physics) and the strength of SNPA (to control long-range nuclear correlations), as we do in MEEFT.

\section{$3.5 \mu-{ }^{3} \mathrm{He}$ capture}

Ackerbauer et al. [69] carried out a high-precision measurement of the capture rate for $\mu^{-} \mathrm{He}^{3} \rightarrow \nu_{\mu} t$. Achieving astonishing accuracy of $0.3 \%$, they reported the experimental value $\Gamma\left(\mu^{3} \mathrm{He}\right)^{\exp }=1494(4) \mathrm{s}^{-1}$. A detailed MEEFT calculation of this capture rate was performed by Gazit [70]. Here again, the only unknown LEC, $\widehat{d}_{R}$, was fixed by fitting to $\Gamma_{\beta}^{t}$ for each given value of the cutoff parameter $\Lambda_{N N}$ (ranging $500 \sim 800 \mathrm{MeV})$, and then $\Gamma\left(\mu^{3} \mathrm{He}\right)$ was calculated. The results are summarized as $\Gamma\left(\mu^{3} \mathrm{He}\right)=1499(2)_{\Lambda}(3)_{N M}(5)_{t}(6)_{R C}$, where the first error comes from the $\Lambda_{N N}$ dependence, the second error from uncertainties in extrapolating the nucleon weak form factors to finite momentum transfer, and in the choice of specific nuclear models, the third error from experimental accuracy for $\Gamma_{\beta}^{t}$, and the fourth error from uncertainty in radiative corrections. The theoretical value (with total error $\sim 1 \%$ ) agrees well with $\Gamma\left(\mu^{3} \mathrm{He}\right)^{\exp }$, providing a yet another case to support the validity of MEEFT; see also Gazit et al. [71], where a full EFT calculation for tritium $\beta$-decay and the tritium binding energy is presented.

In Ref. [59], quoted earlier in connection to $S_{p p}$ calculations, Marcucci et al. also carried out an elaborate calculation of $\Gamma\left(\mu^{3} \mathrm{He}\right)$ in both SNPA and MEEFT, and they found excellent agreement between the two approaches. Combining the results coming from these two methods, Marcucci et al. arrived at a highly accurate estimate $\Gamma\left(\mu^{3} \mathrm{He}\right)=1484 \pm 13 \mathrm{~s}^{-1}$. Again, the theoretical value agrees very well (within quoted errors) with the above experimental value.

\subsection{Neutron-neutron scattering length $a_{n n}$}

In the construction of a high-precision phenomenological $N N$ potential, $V_{N N}^{\text {phenom }}$, the experimental errors in the $n n$ scattering length, $a_{n n}$, are embedded in the overall $\chi^{2}$ value, and it is not obvious to what extent the existing samples of $V_{N N}^{\text {phenom }}$ directly reflect the uncertainties in $a_{n n}$. Meanwhile, low-energy electroweak transitions in lightest nuclei are in general rather sensitive to the precise value of $a_{n n}$. For instance, in [4], uncertainty in the $p p$-fusion $S$-factor, $S_{p p}(0)$, due to the existing uncertainty in

a rather "esoteric" quantity, introduced just to "dramatize" the correlation between $\sigma_{\text {Hen }}$ and binding properties of the relevant nuclei. The message here is that, to get a reliable estimate of $\sigma_{\text {Hen }}$, one must employ, even among so-called high-precision nuclear potentials, only those that can reproduce the binding properties of the $A=3$ and 4 nuclei with needed accuracy. This criterion does not require the introduction of $\zeta$. 
$a_{n n}$ is estimated to be $0.5 \%$, which is not devastatingly large but rather significant. Apart from this point, it is of general importance to determine, as accurately as possible, the empirical value of $a_{n n}$, one of the fundamental parameters characterizing the $N N$ interaction. Since extraction of $a_{n n}$ from the analysis of $n d \rightarrow n n p$ reaction at present involves some inconsistencies [72, 73, 74], the standard value $a_{n n}=-18.63 \pm 0.27(\exp ) \pm 0.30$ (theor) fm [75] is based on the $\pi^{-} d \rightarrow \gamma n n$ reaction. The theoretical framework responsible for this result uses phenomenological $N N$ interactions, and intrinsic short-distance physics uncertainties in this framework limit the theoretical precision. Gardestig and Phillips [76, 77] investigated the possibility of using EFT to reduce theoretical uncertainties in the extraction of $a_{n n}$. They have shown that short-range physics involved in the reaction can be parameterized using the same LEC, $\widehat{d}_{R}$, as introduced in [31], and that, if for the sake of demonstration the currently accepted value of $\widehat{d}_{R}$ is used, the theoretical uncertainty in $a_{n n}$ can be reduced down to $\Delta a_{n n}$ (theor) $=0.05 \mathrm{fm}$. This is quite an impressive reduction (by a factor of 6 ) of the existing theoretical uncertainty.

\subsection{Radiative corrections}

At the level of a few percent precision, radiative corrections (RCs) become an important issue. For instance, in $\mu p$ capture, the electroweak $\mathrm{RC}$ in the semileptonic reaction and the QED corrections to the muonic atom wave function are found to increase the capture rate by $2.8 \pm 0.4 \%$ [78]. The best available estimates of RCs for single-nucleon processes have been given by Marciano and Sirlin [79] for neutron $\beta$ decay, and by Czarnecki et al. [78] for $\mu p$ capture. In these works RCs of order $\alpha$ is decomposed into so-called outer and inner corrections [80]. The outer correction is a universal function of the lepton energy and is independent of the details of hadron physics, whereas the inner correction is affected by short-distance physics and the hadron structure. The inner corrections coming from $\gamma$-(weak boson) loop diagrams are divided into high-momentum and low-momentum parts. The former is calculated in the current quark picture, while the latter is evaluated with the use of the phenomenological weak form factors of the nucleon. As regards RCs in multinucleon systems, all existing works (except those related to the $0^{+} \rightarrow 0^{+}$Fermi transitions), concentrate on the single-nucleon RC diagrams because the genuinely two-bodytype RCs are expected to be small. Kurylov et al. [81] carried out extensive studies of RCs in the $A=2$ systems based on the Marciano-Sirlin approach [79], and their results have been widely used in analyzing the $A=2$ weak-interaction observables (including those discussed earlier in this article). According to [81], with the use of $G_{F}$ from $\mu$-decay, and the "effective" $g_{A}$ obtained from neutron $\beta$-decay, radiative corrections specific to $p p$-fusion is estimated to be $\sim 3-4 \%$ (with $0.1 \%$ uncertainty).

Although the existing estimates of RCs based on the Marciano-Sirlin approach [79] are believed to be reliable at the level of quoted accuracy, it is worth noting that $\mathrm{HB} \chi \mathrm{PT}$ offers a model-independent framework to calculate RCs, insofar as all the relevant LECs are known. The first $\mathrm{HB} \chi \mathrm{PT}$ evaluation of $\mathrm{RC}$ for neutron $\beta$ decay was carried out to order $\alpha$ by Ando et al. [82]. In this approach the short- 
distance physics contributions can be condensed into two LECs, one related to the Fermi constant $G_{F}$ and the other to the axial coupling constant $g_{A}$. If one can determine these LECs from empirical data, one would have a totally model-independent way to evaluate RCs to neutron $\beta$ decay. A similar situation is expected to exist for any low-energy weak-interaction processes on a single nucleon. Even if one cannot determine these LECs at present, it seems of great interest to correlate RCs for various weak processes on a single nucleon taking advantage of the fact that we know exactly what LECs are involved.

\section{Summary}

Despite the limited scope of topics covered, we hope we have succeeded in conveying the message that MEEFT is a highly reliable predictive framework for computing transition amplitudes for a large class of electroweak processes in light nuclei. We remark that, in each of the cases for which both SNPA and MEEFT calculations have been performed, it has been found that the results of the two methods agree very well.

Although MEEFT has been registering and will continue to score significant successes, we should mention that great progress is being made in attempts to carry out full EFT (or "AB-EFT") calculations in which both the transition operators and nuclear wave functions are derived from $\mathrm{HB} \chi \mathrm{PT}$; see. e.g., [83, 84, 85]. We believe that, as increasingly higher order terms are included in AB-EFT calculations, their results will come increasingly closer to those of MEEFT, which are anchored on accurate experimental inputs. Although the developments in AB-EFT calculations are enormous and highly impressive, we will not discuss them here; we trust they will be covered in Ulf Meissner's contribution to this volume.

As mentioned, Riska and Brown's ground-breaking work on the $n p \rightarrow \gamma d$ reaction almost three decades ago was a precursor of explosive developments in high-precision calculations of electroweak processes in light nuclei. We hope Gerry is happy to see the enormous growth of the field in which he has played such an important role.

\section{Acknowledgment}

It is our great pleasure to contribute this article to the Festschrift in honor of Gerry Brown's 85th birthday. A detailed account of MR's life-long scientific collaboration with Gerry is given in another article contributed to this volume. KK started to work with Gerry Brown in 1986, when Gerry kindly invited him to spend a sabbatical year at Stony Brook. KK wishes to express his deepest gratitude to Gerry for having been an inexhaustible source of inspiration and encouragement for him ever since. This article is largely based on the work done in collaboration with Tae-Sun Park, to whom we owe our sincerest thanks. The work of KK is partially supported by the US National Science Foundation under grant number 
PHY-0758114 and that of MR by the WCU Project of the Korean Ministry of Education, Science and Technology (R33-2008-000-10087-0).

\section{References}

[1] D.-O. Riska and G.E. Brown, Phys. Lett. B 38, 193 (1972).

[2] M. Chemtob and M. Rho, Nucl. Phys. A 163, 1 (1971).

[3] See, e.g., J. Carlson and R. Schiavilla, Rev. Mod. Phys. 70, 743 (1998).

[4] E. Adelberger et al., "Solar fusion cross sections II: pp-chain and CNO cycle", arXiv:1004.2314 (to appear in Rev. Mod. Phys.).

[5] V.A. Kuzmin, Phys. Lett. 17, 27 (1965).

[6] S. Fukuda et al., Phys. Rev. Lett. 86, 5651 (2001).

[7] Q. Ahmad et al., Phys. Rev. Lett. 87, 071301 (2001); Phys. Rev. Lett. 89, 011301 (2002); Phys. Rev. Lett. 89, 011302 (2002); B. Aharmim et al., Phys. Rev. Lett. 101, 111301 (2008).

[8] J. Bahcall, M. Pinsonneault and S. Basu, Astrophys. J. 555, 990 (2001).

[9] T. Yamazaki et al. (RBC-UKQCD Collaborations), Phys. Rev. D 79, 114505 (2009).

[10] E. Ivanov and E. Truhlik, Nucl. Phys. A 316, 451,437 (1979).

[11] S. Weinberg, Phys. Lett. B 251, 288 (1990); Phys. Lett. B 295, 114 (1992).

[12] V. Bernard, N. Kaiser and U.-G. Meissner, Int. J. Mod. Phys. E 4, 193 (1995).

[13] U. van Kolck, Prog. Part. Nucl. Phys. 43, 337 (1999).

[14] G.P. Lepage, in: Proceedings of the Second Workshop on Effective Field Theories in Nuclear Physics, eds. P. Bedaque et al., (World Scientific, 1999), p. 353.

[15] S. Beane et al., in "At the Frontier of Particle Physics - Handbook of QCD", ed. M. Shifman, vol. 1 (World Scientific, Singapore, 2001).

[16] G.E. Brown and M. Rho, Phys. Rep. 363, 85 (2002).

[17] S. Weinberg, Physica 6A, 327 (1979).

[18] See, e.g., H. Georgi, Weak Interactions and Modern Particle Physics (Benjamin, 1984).

[19] J. Gasser and H. Leutwyler, Ann. Phys. 158, 142 (1984); J. Gasser, M. Sainio and S. Švarc, Nucl. Phys. B 307, 779 (1988). 
[20] M. Rho, Phys. Rev. Lett. 66, 1275 (1991).

[21] T.-S. Park, D.-P. Min and M. Rho, Phys. Rep. 233, 341 (1993).

[22] T.-S. Park, D.-P. Min and M. Rho, Phys. Rev. Lett. 74, 4153 (1995).

[23] D. Kaplan, M. Savage and M. Wise, Nucl. Phys. B 478, 629 (1996); Nucl. Phys. B 535, 329 (1998).

[24] P.F. Bedaque, H.-W. Hammer amd U. van Kolck, Phys. Rev. Lett. 82, 463 (1999).

[25] J.-W. Chen, G. Rupak and M.J. Savage, Nucl. Phys. A 653, 386 (1999).

[26] T.-S. Park, K. Kubodera, D.-P. Min and M. Rho, Phys. Rev. C 58, 637 (1998).

[27] T.-S. Park, K. Kubodera, D.-P. Min and M. Rho, Astrophys. J. 507, 443 (1998).

[28] T.-S. Park, K. Kubodera, D.-P. Min and M. Rho, Nucl. Phys. A 646, 83 (1999).

[29] T.-S. Park, K. Kubodera, D.-P. Min and M. Rho, nucl-th/9904053; M. Rho, in: Proceedings of the Second Workshop on Effective Field Theories in Nuclear Physics, ed. P. Bedaque, M. Savage, R. Seki, U. van Kolck (World Scientific, Singapore, 1999), p. 225.

[30] T.-S. Park, K. Kubodera, D.-P. Min and M. Rho, Nucl. Phys. A 684, 101c (2001).

[31] T.-S. Park, L. Marcucci, R. Schiavilla, M. Viviani, A. Kievsky, S. Rosati, K. Kubodera, D.-P. Min and M. Rho, Phys. Rev. C 67, 055206 (2003).

[32] L. Marcucci et al., Phys. Rev. Lett. 84, 5959 (2000); L. Marcucci, R. Sciavilla, M. Viviani, A. Kievsky, S. Rosati and J. Beacom, Phys. Rev. C 63, 015801 (2001).

[33] S. Ando, Y.H. Song, T.-S. Park, H. Fearing and K. Kubodera, Phys. Lett. B 555, 49 (2003).

[34] R. Schiavilla et al., Phys. Rev. C 58, 1263 (1998).

[35] J. Carlson, D.-O. Riska, R. Schiavilla and R.B. Wiringa, Phys. Rev. C 44, 619 (1991).

[36] X. Kong and F. Ravndal, Phys. Rev. C 64, 044002 (2001).

[37] M. Butler and J.-W. Chen, Phys. Lett. B 520, 87 (2001).

[38] S. Ando et al., Phys. Lett. B 668, 187 (2008).

[39] K. Kubodera and S. Nozawa, Int. J. Mod. Phys. E 3, 101 (1994). 
[40] S. Nakamura, T. Sato, V. Gudkov and K. Kubodera, Phys. Rev. C 63, 034617 (2001).

[41] S. Nakamura, T. Sato, S. Ando, T.-S. Park, V. Gudkov, F. Myhrer and K. Kubodera, Nucl. Phys. A 707, 561 (2002).

[42] M. Butler and J.-W. Chen, Nucl. Phys. A 675, 575 (2000); M. Butler, J.-W. Chen and X. Kong, Phys. Rev. C 63, 035501 (2001).

[43] M. Butler, J.-W. Chen and P. Vogel, Phys. Lett. B 549, 26 (2002).

[44] J.-W. Chen, K.M. Heeger and R.G.H. Robertson, Phys. Rev. C 67, 025801 (2003).

[45] D.R. Phillips and T.D. Cohen, Nucl. Phys. A 668, 45 (2000).

[46] S. Bogner, T.T.S. Kuo and L. Corragio, Nucl. Phys. A 684, 432c (2001); S. Bogner, T.T.S. Kuo, L. Corragio, A Covello and N. Itaco, Phys. Rev. C 65, 051301 (2002); A. Schwenk, G.E. Brown and B. Friman, Nucl. Phys. A 703, 745 (2002).

[47] B. Mosconi, P. Ricci, E. Truhlik and P. Vogel, Phys. Rev. C 75, 044610 (2007).

[48] S. Ando, T.-S. Park, K. Kubodera and F. Myhrer, Phys. Lett. B 533, 25 (2002).

[49] V.A. Andreev et al. (MuSun Collaboration), arXiv:1004.1754.

[50] P. Kammel and K. Kubodera, Annu. Rev. Nucl. Part. Sci. 60, 327 (2010).

[51] V. Bernard, N. Kaiser and U.-G. Meissner, Phys. Rev. D 50, 6899 (1994).

[52] V.A. Andreev et al., Phys. Rev. Lett. 99, 032002 (2007).

[53] N. Tatara, Y. Kohyama and K. Kubodera, Phys. Rev. C 42, 1694 (1990).

[54] J. Adam et al., Nucl. Phys. A 507, 675 (1990).

[55] P. Ricci, E. Truhlik, B. Mosconi and J. Smejkal, Nucl. Phys. A 837, 110 (2010).

[56] J.-W. Chen, T. Inoue, X.-D. Ji and Y.-C. Li, Phys. Rev. C 72, 061001 (2005).

[57] G. Bardin et al., Nucl. Phys. A 453, 591 (1986).

[58] M. Cargnelli et al., Proc. Yamada Conf. Nucl. Weak Process Nucl. Struc., 23rd, p.115 (1989).

[59] L.E. Marcucci et al., arXiv:1008.1172 [nucl-th].

[60] D.R. Entem and R. Machleidt, Phys. Rev. C 68, 041001 (2003).

[61] C. Werntz and J.G. Brennan, Phys. Rev. 157, 759 (1967). 
[62] K. Kubodera and T.-S. Park, Annu. Rev. Nucl. Part. Sci. 54, 19 (2004).

[63] F.L.H. Wolfs et al., Phys. Rev. Lett. 63, 2721 (1989).

[64] R. Wervelman et al, Nucl. Phys. A 526, 265 (1991).

[65] R. Lazaukas, Y.-H. Song and T.-S. Park, "Heavy-baryon perturbation theory approach to thermal neutron capture on ${ }^{3} \mathrm{He}$ ", arXiv:0905.3119 [nucl-th].

[66] O.A. Yakubowsky, Sov. J. Nucl. Phys. 5, 937 (1967).

[67] Y.-H. Song, R. Lazaukas and T-S. Park, Phys. Rev. C 79, 064002 (2009).

[68] T.-S. Park, K. Kubodera, D.-P. Min and M. Rho, Phys. Lett. B 472, 232 (2000).

[69] P. Ackerbauer et al., Phys. Lett. B 417, 224 (1998).

[70] D. Gazit, Phys. Lett. B, 666, 472 (2008),

[71] D. Gazit, S.Quaglioni and P. Navrátil, Phys. Rev. Lett. 103, 102502 (2009).

[72] V. Huhn et al., Phys. Rev. C 63, 014003 (2000).

[73] W. van Witch, V. Ruan and H. Witala, Phys. Rev. C 74, 014001 (2006).

[74] D.E. Gonzales Trotter et al., Phys. Rev. C 73, 034001 (2006).

[75] Q. Chen et al., Phys. Rev. C 77, 054002 (2008).

[76] A. Gardestig and D.R. Phillips, Phys. Rev. C 73, 014002 (2006); A. Gardestig and D.R. Phillips, Phys. Rev. C 74, 017001 (2006).

[77] A. Gardestig, J. Phys. G 36, 053001 (2009).

[78] A. Czarnecki, W.J. Marciano and A. Sirlin, Phys. Rev. Lett. 99, 032003 (2007).

[79] W.J. Marciano and A. Sirlin, Phys. Rev. Lett. 56, 22 (1986).

[80] A. Sirlin, Phys. Rev. 164, 1767 (1967); A. Sirlin, Rev. Mod. Phys. 50, 573 (1978).

[81] A. Kurylov, M.J. Ramsey-Musolf and P. Vogel, Phys. Rev. C 67, 035502 (2003).

[82] S. Ando et al., Phys. Lett. B 64, 250 (2004).

[83] E. Epelbaum, H.-W. Hammer and U.-G. Meissner, Rev. Mod. Phys. 81, 1773 (2009).

[84] S. Kölling et al., Phys. Rev. C 80, 045502 (2009).

[85] D. Rozpedzik, J. Golak, S. Kölling and E. Epelbaum, "Two-pion exchange currents in photodisintegration of the deuteron," arXiv:1010.6269 [nucl-th]. 